Théologiques

Théologiques

\title{
Le Zarathoustra de Nietzsche et Maître Eckhart
}

\section{Nishitani Keiji}

Volume 20, numéro 1-2, 2012

Les philosophes de l'École de Kyōto et la théologie

URI : https://id.erudit.org/iderudit/1018858ar

DOI : https://doi.org/10.7202/1018858ar

Aller au sommaire du numéro

\section{Éditeur(s)}

Faculté de théologie et de sciences des religions, Université de Montréal

\section{ISSN}

1188-7109 (imprimé)

1492-1413 (numérique)

Découvrir la revue

\section{Citer cet article}

Keiji, N. (2012). Le Zarathoustra de Nietzsche et Maître Eckhart. Théologiques, 20(1-2), 169-198. https://doi.org/10.7202/1018858ar

\section{Résumé de l'article}

Les pensées de Nietzsche et de Maître Eckhart constituent deux influences majeures qui traversent toute l'oeuvre de Nishitani. Dans ce beau texte, rédigé lors de son séjour d'études (1937-1939) à l'université de Fribourg auprès de Heidegger, Nishitani fait une lecture tout à fait originale et innovante de la mystique eckhartienne, en la mettant en contraste avec la figure de Zarathoustra chez Nietzsche. Au-delà de leurs conditions historiques et de la spécificité de leurs démarches respectives, il leur reconnaît une attitude fondamentale commune qui consiste à approfondir ce qu'il nomme le " mouvement dialectique de la vie " (lequel vise une affirmation de l'homme à travers la négation de celui-ci) en direction du fond infondé qui soutient toute existence. Par " caractère originaire de la vie ", il entend la source de la vie dans son absence de fondement et de limite, dans son absence de raison. $\mathrm{Ce}$ faisant, et bien que relativement précoce (1938), cet essai annonce quelques-unes des intuitions les plus fulgurantes de Nishitani et offre une première esquisse de la plupart des thématiques qui lui tiendront particulièrement à coeur dans ses travaux à venir. Aussi y est-il déjà question de la vacuité, du savoir du non-avoir par opposition à la distinction sujet-objet, de la problématique de la représentation attachée à son exigence d'objectivité, de la compréhension de l'au-delà comme immanent à ce monde-ci, etc. Même la thématique du nihilisme, qui n'est pourtant pas nommément mentionnée, y fait écho dans l'exemple significatif du sentiment que tout est égal, que tout se vaut. En raison de la forme encore largement germinale que prennent ici ces réflexions, il est plus aisé d'en percevoir la portée et les imbrications. D’une certaine manière, on pourrait considérer cet essai comme un prélude aux importants développements que connaîtra la pensée nishitanienne jusqu'à l’ouvrage de maturité, Qu'est-ce que la religion? ?(『宗教とはなにか』) de 1961.
Ce document est protégé par la loi sur le droit d'auteur. L'utilisation des services d’Érudit (y compris la reproduction) est assujettie à sa politique d'utilisation que vous pouvez consulter en ligne.

https://apropos.erudit.org/fr/usagers/politique-dutilisation/ 


\title{
Le Zarathoustra de Nietzsche et Maître Eckhart $^{1}$
}

\author{
Nishitani Keiji \\ Traduit par Sylvain IsAAC
}

\section{Le caractère originaire ${ }^{3}$ de la vie chez Nietzsche}

Après s'être adressé au soleil qui, à l'aurore, montait de la vallée, Zarathoustra descendit seul de sa montagne, de retour vers le monde des

1. Traduction de Nishitani, K. (1988), ニイチェのツァラツストラとマイスター・ エックハルト, dans 『西谷啓治著作集』(Euvres de Nishitani Keiji), vol. 1: 根源 的主體性の哲学・正 (Philosophie de la subjectivité originaire (début)), Tōkyō, Sōbunsha, p. 5-32. Cet essai, rédigé en juin 1938 lors du séjour d'études de Nishitani en Allemagne, fut publié pour la première fois en septembre de la même année dans Ishihara (1938, 327-366).

2. Ce chiffre et les suivants dans le corps du texte renvoient à la pagination du texte original. Les notes de bas de page sont du traducteur. Les références données dans le corps du texte sont de Nishitani. Le lecteur trouvera, en fin de volume, un glossaire français-japonais rassemblant les termes clés que l'on rencontre chez Nishitani et certains autres philosophes de l'École de Kyōto.

3. Après mûres réflexions, nous avons choisi de traduire 根源的 (kongenteki) par "originaire ». Jusqu'à présent, et à la suite des traductions anglaises de Nishitani, ce terme était généralement rendu par «élémental» (vocable qui fut introduit en 1982 lors de la parution du Religion and Nothingness de Nishitani afin de faire écho à la fois à la quête des éléments premiers chez les présocratiques et à l'Elementalphilosophie de K. L. Reinhold; voir Heisig 2008, 416). Outre le fait que ce néologisme n’est ni très élégant ni guère parlant en français, il ne rend pas non plus précisément la signification du terme japonais. Le premier kanji (根) signifie racine, origine. Le second (源) signifie source, origine. Sous la plume nishitanienne, ce terme désigne le plan originel indéterminé et non substantiel d'où surgit la vie. Plutôt que «séminal » ou « racinal» (qui s'en rapprochent), nous avons opté pour "originaire ", ce qui est en conformité avec le choix fait par Bernard Stevens dans sa propre traduction du Qu'est-ce que la religion? de Nishitani, dont le lecteur trouvera le premier chapitre dans les pages de ce volume.

(C) Revue Théologiques 2012. Tout droit réservé. 
hommes. Il rencontra un vieux saint qui, vivant retiré dans la forêt au pied de la montagne, louait Dieu. Lorsqu'il quitta le saint homme, Zarathoustra parla ainsi à son cœur: "Serait-ce donc possible? Ce vieux saint dans sa forêt, il ne l'a donc pas encore appris que Dieu est mort! » Ce faisant, il arriva en ville.

Ce passage du prologue annonce l'esprit dans lequel se développera l'ensemble du Zarathoustra. Jadis, Zarathoustra avait déjà traversé cette forêt. Il était alors mort au monde des hommes. Le saint homme dit: «Il y a bien des années, Zarathoustra passa par ici [...]. Tu portais alors tes cendres à la montagne». Or, dix ans durant, au sommet des montagnes, Zarathoustra "se délecta de son esprit et de sa solitude ", et son cœur changea. Les cendres froides se transformèrent en vie débordante. Cette vie l'incita à redescendre vers le monde des hommes. La forêt se trouve là où commence la montagne qui s'élève au-dessus du monde des hommes. Le saint homme y vit tel "un ours parmi les ours, un oiseau parmi les oiseaux ». Fuyant la compagnie des hommes, il se tient auprès de Dieu : "Je compose des chants et je les chante, et tout en composant mes chants, je ris, je pleure et je grommelle: c'est ainsi que je loue Dieu. - Chantant, riant, pleurant et grommelant, je loue Dieu, ce Dieu qui est mon Dieu ». /6/ Nourri au sein de Dieu, il jouit d'une paisible innocence semblable à celle d'un enfant.

Il y a, au plus profond de la vie humaine, une innocence dans laquelle bien et mal sont encore indistincts et d'où ne germe aucune malveillance. Bien que la vie humaine y trouve son origine, elle se gonfle bientôt de malveillance et est précipitée dans les mondes tourbillonnants d'Avīci et de Raurava ${ }^{4}$. Seulement chez le nourrisson se reflète encore imperceptiblement cette origine de la vie. Ce n'est qu'en étant semblable au nourrisson que l'on peut entrer au Paradis. Le vieux saint est redevenu semblable au nourrisson: il est devenu un saint homme. Le saint homme se distingue en ceci qu'il est revenu à la naturalité originelle.

L'homme, bien qu'il soit un être naturel issu de la nature au même titre que les pierres, les plantes et les animaux, s'enivre de l'atmosphère chaleureuse et braillarde des relations humaines, de la «société ", et oublie sa propre naturalité. Lorsque, chaussé, il marche sur une route goudronnée, l'homme oublie qu'il a lui-même jailli de ce sol. Couvrant son corps de vêtements, il oublie qu'il y a une profondeur insondable au fond de son soi

4. Avīci (jap. abi) et Raurava (jap. kyōkan) sont deux des huit grands enfers brûlants dans la cosmologie bouddhique. 
dépouillé. S'entourant de quatre murs, il oublie que le ciel infini l'englobe et englobe aussi le toit qui l'en sépare. Ne sont-ils pas rares ceux qui, à partir du morceau qui se trouve dans leur assiette, se souviennent de la forme du poisson qui nageait dans le Kuroshio ${ }^{5}$ ? Plus rares encore sont ceux qui s'aperçoivent qu'au fond une vie commune anime ce poisson et eux-mêmes.

Personne n'a encore rédigé de "philosophie du vêtement", mais on pourrait dire que l'origine de tous les maux réside dans le fait d'avoir couvert le corps. L'habit rend nécessaire le miroir. Depuis que les premiers êtres humains ont enduit leurs visages d'argile et se sont mirés dans un miroir d'eau, les hommes ont perdu le soi affleurant directement au soi, et ils ont pris l'habitude irrépressible de s'appréhender eux-mêmes à travers le soi qui se reflète dans l'œil d'autrui. Le miroir, c'est l'œil d'autrui dans toute sa généralité. Ce que les autres peuvent voir, ce qu'ils en peuvent penser, s'est substitué à «l'être » naturel et a fini par constituer l'existence du soi. L' «être» apparent ${ }^{6}$ s'est propagé jusque dans les profondeurs de l'âme; à l'instar du corps enveloppé dans un vêtement, l' «être » naturel de l'âme est englobé par cet «être» apparent. Les hommes appréhendent, en eux-mêmes, un soi imaginaire qui n'a rien de réel. Et, au moyen des fils innombrables tissés à partir de ce simulacre d'existence, ils se lient les uns aux autres et constituent des sociétés qui ont divorcé d'avec la nature. Dans ce monde aliéné, ils s'oublient dans un soi imaginaire et, en tant qu'êtres rationnels, manipulent à leur guise un soi devenu présomptueux ( « eingebildet»).

Échapper à cette "ville» trop humaine /7/, c'est reconduire à son origine la raison qui s'est écartée de la naturalité et la transformer en une manifestation de la vie naturelle, au même titre que l'instinct animal. Il s'agit de rabaisser la raison en même temps que de l'élever. Il s'agit de nier la superbia (l'arrogance) de la raison et de revenir à la vie profonde. Il s'agit de reconduire la raison qui, tombée sous l'emprise de l'imagination, n'est plus qu'une simple Bild (ou forme imagée) dénuée de toute réalité à sa véritable réalité comprise comme ce qui affranchit du joug de l'imagi-

5. Courant maritime qui longe les côtes du Japon, à la manière du Gulf Stream le long des côtes européennes.

6. Jeu de mot de Nishitani autour du terme mie (見え). Composé de deux kanjis (見栄), ce terme signifie "vanité», "esbroufe». Mais en remplaçant le second kanji par l'hiragana え, il fait en même temps allusion à l'être «que peut voir» (見える) autrui dont il est question dans la phrase précédente. Nous avons choisi de rendre cette polysémie par le terme «apparence». 
nation et qui se manifeste depuis la profondeur insondable de la vie, comprise comme ce qui a jailli de la terre.

Dans ce moment-là, même l'activité intellectuelle en vient à ressembler aux chants des oiseaux ou aux grommellements de l'ours; elle s'apparente aux rides qui ondulent paisiblement à la surface de la mer de la vie divine, de la vie sans fond au fond de la nature. La vie infinie s'accomplit même dans les pleurs et les rires. Les pleurs et les rires aussi sont des louanges adressées à Dieu. La sainteté se révèle lorsque l'on meurt au monde humain trop humain et que l'on revient à sa nature profonde. Alors, les grands bâtiments en enfilade se confondent avec des arbres alignés, et l'homme qui bavarde ressemble à l'oiseau qui chante. Celui que rencontra Zarathoustra était un saint homme vivant ainsi dans la forêt et fuyant la ville.

Cela dit, la forêt ne se situe qu'à la moitié du chemin. La logique de Hegel lui reconnaît toutefois un statut: la négation doit à nouveau être niée. Il s'agit là d'une nécessité vitale avant d'être une nécessité logique. C'est une exigence qui surgit des profondeurs de la vie. Le saint homme, en mourant au monde des hommes, a obtenu l'union avec la vie divine au sein de la nature. Si l'on se place du point de vue de cette vie nouvelle, la vie dans le monde des hommes s'apparente à la mort. Toutefois, aussi haute soit-elle, cette vie nouvelle maintient sa propre source hors d'ellemême. Cette vie, se tenant devant Dieu, fait deux avec lui. Trouver refuge en Dieu, c'est se séparer de Dieu.

Il est clair que ce n'est pas en entendant la parole de la grâce divine dans la prise de conscience aiguë de sa propre culpabilité que ce saint homme s'est élevé à Dieu. Par conséquent, il n'a rien à voir avec la Koketterie, l'arrogance "humble ", qui consiste à s'engager intentionnellement dans l'hérésie inhérente à cette voie et à vouloir payer chèrement l'amour divin. Toutefois, dans la mesure où il objective Dieu, il se tient distinct de la vie divine, et celle-ci ne lui apparaît nullement comme une vie authentique. Que la vie soit perçue en tant qu'objet signifie en réalité la mort de la vie /8/. Et donc, comme la vie qu'il a regagnée est tout à la fois unie à la vie divine et distincte de celle-ci, elle est vie tout en étant encore la mort. Simultanément, parce qu'il fait face à Dieu, il se détourne de l'humanité. Pour lui, la vie humaine s'apparente à la mort. Parce que sa vie se perd dans celle de Dieu, il en oublie le chemin qui mène à la vie humaine. C'est parce qu'il jouit d'une vie d'un plus haut degré qu'il se situe justement au niveau de la mort de Dieu, de la mort du soi et de celle des hommes. En portant le visage de la mort comme son double, sa vie est 
elle-même la mort. Il s'agit là de la vie dans la forêt, laquelle se situe à michemin entre la ville et la montagne. Et le saint homme qui vit reclus en cette forêt ne se rend pas compte que c'est dans la vie d'un plus haut degré, qui est la sienne, que se dissimule la mort de toutes choses.

Zarathoustra, plutôt que de déposer ses cendres froides dans la forêt, a dû traverser celle-ci et attendre, au sommet des montagnes, une métamorphose plus haute, une négation de la négation de la vie. Comme il est bien connu, Plotin a expliqué autrefois que l'âme s'unit à Dieu lorsqu'elle voit le monde intelligible au-dessus du monde sensible et lorsqu'elle a surmonté celui-ci en celui-là. Et, en vertu de ce monde intelligible que l'on considère généralement comme l'endroit où l'âme peut vivre en paix, il a insisté sur la nécessité, pour celui qui continue de voir Dieu comme objet de pensée et reste à la fois identique et distinct, de s'élever plus haut encore vers l'un pur. Il exprima cela en parlant d'extase, d'éveil, du Bien par excellence dont on ne peut pas même dire qu'il est bon ${ }^{7}$, d'un par-delà bien et mal, d'un au-delà de la pensée et de l'être, de volonté, de vie, etc. L'image de Zarathoustra qui quitte la forêt pour poursuivre vers les sommets, ne pourrait-on pas dire qu'elle évoque quelque part l'âme plotinienne qui parcourt les étapes successives des hypostases?

"Zarathoustra est transformé, Zarathoustra est devenu enfant, Zarathoustra est un homme éveillé », s'écrie le saint homme. Quel est le sens de cette métamorphose? Se tournant vers le soleil, Zarathoustra commence à parler: "Bénis ce calice prêt à déborder, que l'eau s'en écoule dorée et qu'elle porte partout le reflet de ton allégresse.» Cette métamorphose révèle une volonté de débordement. Contrairement au saint homme, chez qui le soi est rempli par la vie, c'est la vie qui en vient à remplir le soi. Quand on dit que le soi est rempli, l'aspect du soi se maintient. Le soi reste forme. Dans ce cas, l'eau de la vie se contente de remplir l'espace du calice. Quand on dit que la vie en vient à remplir le soi, l'eau de la vie /9/ déborde et brise l'aspect du calice. La forme du soi, qui persistait bien que devenue vacuité, est rejetée depuis l'intérieur. Le soi n'est déjà plus une "substance». Ce n'est pas non plus le centre déterminé des actes de conscience. C'est quelque chose qui peut appréhender une forme infinie sans forme déterminée.

"Cet homme, ne marche-t-il pas tel un danseur? ", s'interroge le saint homme. En vérité, dans ses propres sermons, Zarathoustra lui-même exhorte souvent: "Apprenez à danser». Sans s'attacher à une forme, il

7. Ennéades (V, 5, 13). 
s'agit de se convertir à une vie de danse, laquelle est un flux où les formes s'interpénètrent mutuellement. Dans cette volonté de débordement - où l'on pourrait voir également un reliquat de l'âme extatique dont parle Plotin, ou de la volonté de l'un «d'émaner» —, même la dévotion à Dieu qui habitait le saint homme s'estompe. Plutôt que deux volontés qui s'unifient, il n'y a qu'une volonté, la volonté de Zarathoustra qui s'est défait de la forme du soi. Dans cette volonté une, la vie qui, chez le saint homme, persistait à englober la mort au plus haut point devient une vie authentiquement vécue. Autrement dit, elle devient une vie créative. Et, à l'instar de l'eau dorée qui, ayant débordé du calice, s'écoule vers le bas, cette vie doit s'orienter vers le bas. La création doit inévitablement devenir un déclin (Untergang). Le calice est rempli d'eau jusqu'à ras bord. Zarathoustra s'est lassé de la perfection de sa sagesse. Lorsqu'un fruit est bien mûr, il se détache de l'arbre qui l'a nourri jusque-là et ne peut que retomber sur la terre d'où il était issu. Par la même nécessité vitale, Zarathoustra décide de quitter la montagne et de redescendre vers la ville, de rejoindre la société humaine. Il proclame: «Ce calice aspire à se vider à nouveau et Zarathoustra veut redevenir homme».

Il est dit que c'est par amour que la parole de Dieu se fait chair. De la même manière, on peut dire également que c'est par amour que Zarathoustra, qui avait traversé la «sainte» forêt pour se retirer sur les cimes solitaires où il se transforma, est redescendu dans la société. Quand, à son apogée, la vie devient authentiquement créative, elle mûrit la volonté de Untergang (c'est-à-dire de déclin ou de ruine). Devenir authentiquement créatif, c'est devenir authentiquement capable de décliner. La fructification est le point culminant de la vie d'un arbre; en outre, c'est à l'instant précis où le fruit se détache de l'arbre et tombe sur le sol que l'arbre manifeste au plus haut degré son caractère créatif. /10/ Toute véritable création se parachève dans une ruine complète, et seule une vie qui est arrivée à se faire authentiquement créative peut s'achever dans une ruine véritable. C'est là l'ultime essor de la création: à partir du point le plus haut, il s'agit d'accomplir un pas supplémentaire. Voilà la raison pour laquelle l'autonégation absolue de la vie constitue immédiatement son autoaffirmation absolue. C'est là que la mort de toute chose, dont il a été question plus haut, se change en vie de toute chose.

La question se pose de savoir si cet élément apparaît dans la philosophie de Hegel. Sa logique, qui s'attache pourtant à examiner chaque parcelle de la vie, n'aurait-elle pas omis de considérer la limite de la logique au point ultime où la négation de la négation se transforme immédiatement 
en affirmation? Et ne serait-ce pas précisément sur ce point que la perspective du matérialisme historique se différencie et qu'apparaît la possibilité de renverser encore une fois la logique hégélienne et de la corriger? Même si Fries, Schelling, Schopenhauer ont critiqué Hegel dans une perspective qui leur est à chaque fois propre, on peut affirmer qu'en fin de compte ils ont tous dû percevoir cette faiblesse.

Quoi qu'il en soit, il y a là quelque chose d'intéressant: à l'instar des critiques religieux de Hegel, Zarathoustra aussi, chez Nietzsche, s'est efforcé de formuler son état d'esprit au moyen du mot "amour». Toutefois, il renonça aussitôt à cette tentative. Au saint homme qui cherche à le dissuader de redescendre en ville, il déclare: "J'aime les hommes. » Le saint homme répond: «Pourquoi donc suis-je parti dans la forêt et la solitude? N'était-ce pas parce que j'aimais par trop les hommes? - Maintenant, j'aime Dieu. Les hommes, je ne les aime pas. L'homme, pour moi, est une chose par trop imparfaite. L'amour pour l'homme me tuerait.» À travers cet échange, Nietzsche oppose à la volonté de débordement l'insuffisance de l'amour envers Dieu, en raison de son caractère inachevé que nous avons évoqué plus haut (lui qui s'arrête à mi-chemin du mouvement dialectique de la vie). Dans l'achèvement de ce mouvement, il lui oppose toute possibilité d'anéantissement. En entendant la réponse du saint homme, Zarathoustra renonce soudainement à parler d'amour: "Qu'ai-je parlé d'amour, j'apporte aux hommes un présent!» Et au saint homme qui lui dit de ne pas donner aux hommes plus qu'une aumône, il répond: «Non, je ne fais pas d'aumônes. Je ne suis pas assez pauvre pour cela.»

Dans cette réplique ironique /11/, on peut reconnaître un autre aspect du caractère inachevé de l'amour envers Dieu: dans une vie qui ne s'est pas encore faite volonté de débordement, même "l'amour de son prochain" consiste à vouloir suppléer par autrui à notre propre vide intérieur. Ici, l'amour du prochain présuppose une pauvreté en esprit qui, en réchauffant autrui, se réchauffe lui-même. C'est pour cela que Zarathoustra entend "porter le feu dans les vallées ». Ce feu n'est pas destiné à se réchauffer les uns les autres; c'est un feu qui brûle tout un chacun, c'est le feu de la négation absolue. Simultanément à la négation de «l'humanité » en l'homme, c'est-à-dire de ce qui est trop humain, il s'agit aussi de la négation de la dépendance à Dieu. L'homme se raccroche à lui-même sous la forme de l'ego. Ou plutôt, cet attachement à soi est la forme de l'ego comme tel. Mais même dans le cas où l'homme se raccroche à Dieu en se niant luimême, ou en étant nié en Dieu, cet attachement à Dieu comporte une trace d'attachement à soi : celle du «pour-soi». Afin que la négation de l'huma- 
nité soit absolue, il faut aller jusqu'à nier l'humanité incluse dans la dépendance à Dieu. Car lorsque l'humanité est niée, mais qu'elle dépend encore de Dieu, seule la moitié du chemin a été parcourue vers l'absolue négationcomme-affirmation. (La plupart des théologies, y compris la théologie dite «dialectique», en restent ainsi à une semi-dialectique.) Pour cette raison, même l'amour du prochain doit être métamorphosé en une figure nouvelle. L'amour pour notre prochain (c'est-à-dire pour les personnes qui nous sont les plus proches) doit être transformé en un "amour pour les personnes qui nous sont les plus éloignées » : l'affirmation de l'humanité telle qu'elle est immédiatement donnée doit être changée en une affirmation absolument négative de l'humanité. Le feu que Zarathoustra porte dans les vallées est le feu de l'amour pour ces personnes les plus éloignées.

La parole «Dieu est mort » peut donc être comprise, me semble-t-il, à partir de tout ce qui a été dit jusqu'ici. Elle peut être comprise comme ce qui indique l'abîme de la vie, lequel ne se manifeste nullement dans une vie où il est question d' "amour de Dieu ", de «faire face à Dieu» ou encore de «mourir en tant qu'homme et naître en Dieu» ni dans une vie qui s'exprimerait en tant qu'union avec Dieu. Ailleurs, bien que dans un tout autre contexte, Nietzsche affirme: «Si tu regardes longtemps au fond d'un abîme, l'abîme aussi regarde au fond de toi "/12/ (Par-delà bien et mal, $146)$; je pense que c'est la même idée qui transparaît ici. Elle ne réapparaîtra que chez Bergson dans le moment de «l'élan vital », dans le moment de la création. Il va sans dire qu'une telle création se manifeste à l'apogée du mouvement dialectique de la vie et qu'elle ne relève pas d'une vie «simple et immédiate».

Zarathoustra déclare: "Créer, voilà la grande délivrance de la souffrance, voilà ce qui rend la vie légère. Mais pour qu'existe celui qui crée, il faut beaucoup de souffrance et de métamorphose. - Oui, vous qui créez, il faut qu'il y ait beaucoup de morts amères dans votre vie" (II. "Sur les îles bienheureuses»). Dès lors, l'ultime métamorphose requise consiste à surmonter l'ultime démembrement de la vie, c'est-à-dire surmonter le fait de faire face à Dieu, de trouver refuge en Dieu ou de s'unir à lui. C'est la manifestation de «l'un» le plus profond de la vie. «Cette volonté d'engendrer m'attira loin de Dieu et des dieux; qu'y aurait-il donc à créer s'il y avait des dieux?" (idem). "Ah! S'il se trouvait quelqu'un [Dieu] pour sauver les prêtres de leur sauveur» (II. «Des prêtres»).

Déjà auparavant, dans De l'utilité et des inconvénients de l'histoire, Nietzsche opposait «ce qui est éternellement masculin » à «ce qui est éternellement féminin »; ici encore, c'est un esprit identique qui est à l'œuvre. 
«Véridique, c'est ainsi que j'appelle celui qui s'en va dans des déserts d'où Dieu est absent et qui a brisé son cœur qui vénère" (II. "Des sages illustres»). Le cœur qui vénère, c'est le cœur de la foi. D'où Zarathoustra repousse sans cesse celui qui croit en Dieu et le Dieu en lequel il croit. Quant à son attitude vis-à-vis de la foi, elle se fait la plus explicite dans cette parole qu'il adresse à ses disciples: "Vous êtes mes disciples: mais qu'importent tous les disciples! Vous ne vous étiez pas encore cherchés: alors vous m'avez trouvé. C'est ce que font tous les disciples; c'est pourquoi toute foi compte si peu. Maintenant, je vous ordonne de me perdre et de vous trouver; et ce n'est que quand vous m'aurez tous renié, que je veux revenir parmi vous» (I. "De la vertu qui prodigue»). Lorsque celui qui se tient dans la position de la foi, ayant perdu celui en lequel il croit, se cherche et se trouve lui-même, celui en lequel il croyait revient à nouveau. Cela n'indique-t-il pas clairement que le dépassement de la position de la foi /13/ est une entreprise considérable ? C'est la raison même pour laquelle Zarathoustra aime à se présenter comme «impie». "Je suis Zarathoustra, l'impie, celui qui dit: "Qui est plus impie que moi pour que je me réjouisse de me soumettre à son enseignement?" - Je suis Zarathoustra l'impie: où trouverai-je mon semblable, mon égal? Ils sont semblables à moi, ceux qui se donnent eux-mêmes leur volonté et qui se débarrassent de toute résignation» (III. «De la vertu qui rend petit»). Au terme de ce dont il a été question jusqu'ici, il me semble évident que, par essence, cette position se distingue complètement de «l'athéisme » commun.

\section{Le caractère originaire de la vie chez Eckhart}

Au cœur de l'enseignement de Maître Eckhart, mystique allemand du XIII ${ }^{\mathrm{e}}$ siècle, se trouve ce qu'il appelle la naissance de Dieu dans l'âme. Et cette naissance de Dieu signifie devenir purement un avec Dieu. Il ne doit pas seulement s'agir, selon Eckhart, d'entrer dans une " union avec Dieu ", mais d'une authentique manifestation de «l'un» pur. Cette distinction entre l'union et l'un, il la souligne avec insistance à plus d'une occasion: «Ce n'est ni ce qui est enveloppé ni ce qui unifie, c'est l'un»; «Plutôt que de simplement unifier, c'est un unique un (ein einic ein $)^{8}$ "; "Sans rien donner à Dieu ni rien recevoir de Dieu, c'est un Un et une pure unification (ein ein und ein luter einunge $)^{9} »$. Des expressions de ce type sont fréquem-

8. Sermon 23.

9. Sermon 12. 
ment réitérées; elles attestent de son effort pour exprimer une posture qui va jusqu'à dépasser l' «union avec Dieu ».

Dans ce contexte, qu'est-ce qui justifie l'insistance avec laquelle Eckhart souligne cette distinction entre l'union et l'un pur? Selon lui, c'est dans leurs essences ultimes ou, plutôt, c'est précisément au sein même de leurs essences ultimes, que la relation entre Dieu et l'homme est vie de la manière la plus authentique $/ 14$; ; raison pour laquelle leur relation peut être vécue en tant que vie - en tant que vie qui surgit de nos entrailles les plus profondes. Voir en Dieu la vie qu'il faut vivre constitue le fondement de la tradition mystique.

Au cours de la période médiévale de l'Occident, l'augustinisme (qui s'appuyait sur la déclaration augustinienne selon laquelle Dieu est « la vie de toutes les vies ${ }^{10} »$ ) se développa en considérant que la lumière de Dieu se déversait directement dans l'âme humaine et qu'un flux de vie allait de l'un à l'autre. Il s'opposa au penchant intellectualiste qui trouvait son accomplissement chez Thomas d'Aquin (lequel fixait des limites précises au domaine dans lequel peut s'épanouir l'âme humaine et niait tout contact direct avec Dieu), et orienta tout le cours de la mystique médiévale.

Bien que tirant sa source du thomisme, la pensée eckhartienne adopta bientôt le cours de l'augustinisme. Chez Eckhart aussi, la vie est le commencement en même temps que l'achèvement. La vie ne saurait avoir le moindre de ses fondements en dehors d'elle-même. Si l'on posait des fondements transcendants hors de la vie, alors la vie, se surpassant elle-même, ferait siens ces fondements. On ne saurait fixer des limites à la vie. C'est que la vie se fait jour là même où elle franchit ses propres limites. Ou encore, il n'y a aucune raison qui permette d'attacher un sens à la vie. La vie est sans raison. Même si l'on posait une raison aussi haute que possible au-dessus de la vie, la vie s'approprierait cette raison à un niveau plus élevé encore. Elle est ce qui se rend elle-même sans raison. Ce caractère originaire qui est propre à la vie traverse toute la pensée d'Eckhart. Il affirme par exemple ${ }^{11}$ que la vie vit pour vivre, que la vie vit à partir de son propre fond, qu'elle vit sans "pourquoi ", qu'elle se vit elle-même, etc.

En adoptant le réalisme d'Aristote, Thomas d'Aquin a limité le champ du savoir accessible à l'intellect humain au monde des objets sensibles rattachés à la matière. Or, la pensée aristotélicienne reconnaissait en même temps un dynamisme à la vie, il en soulignait le caractère développemental.

10. Dans Les confessions (Livre X, Chapitre 6).

11. Dans le sermon $5 \mathrm{~b}$. 
Et, me semble-t-il, si l'on approfondit cet aspect, la pensée aristotélicienne en arrive inévitablement à manifester un retour au platonisme, lequel situait l'intuition des idées au niveau d'une réalité suprasensible. Eckhart est parti de Thomas d'Aquin, mais il a aussi subi l'influence de l'augustinisme, que l'on peut qualifier de forme médiévale du platonisme. L'influence sur lui de gens comme Bonaventure est attestée par les historiens de la philosophie /15/. Or, pour approfondir le caractère développemental de la vie, on ne saurait se contenter du platonisme. Que le moment dynamique présent dans la pensée aristotélicienne, subsumant de l'intérieur la pensée aristotélicienne elle-même, conduise au platonisme pour ensuite surmonter celui-ci à son tour, nous en avons déjà l'illustration chez Plotin.

Du long fleuve que représente le platonisme, la pensée eckhartienne s'est déversée dans l'abîme du néo-platonisme. Eckhart déclare par exemple: "La justice est vie et consiste à vivre justement; la justice est l'être même en tant que mode d'être»; "L'être de la justice et l'être de celui qui est juste sont un seul et même être " (Käte Otermanns, Meister Eckhart, p. $\left.28^{12}\right)$. De telles affirmations ne peuvent forcément que transcender le platonisme. Chez Platon, la justice comme telle, c'est-à-dire l'idée de la justice, était le modèle et la justice qui se manifeste chez celui qui est juste, une copie. Même Augustin, bien qu'il considérât que la connaissance s'établit là où l'intellect humain voit les idées éternelles, grâce à la lumière divine qui se déverse dans l'âme humaine, ne pouvait se contenter de reconnaître une relation d'imitation ou de ressemblance entre le modèle et la copie. Par ailleurs, quand Eckhart affirme que l'être de la justice et l'être de celui qui est juste ne font qu'un, ce ne peut être qu'en adoptant la position foncière de la vie. S'il peut affirmer que la justice est vie et qu'elle consiste à vivre justement, que la justice est l'être même en tant que mode d'être, c'est uniquement grâce au fait que la relation de la justice et de celui qui est juste est traversée de part en part par la vie. Ce n'est que dans la vie que le modèle et la copie deviennent les deux aspects d'une même réalité. On ne peut affirmer que l'être de la justice et l'être de la personne juste sont le même être que quand, dans la position foncière de la vie, la vie est

12. Nishitani écorne le nom de l'auteure (Oltmanns) et renvoie erronément à la page 28, alors que ces deux citations sont tirées de la page 82. Celles-ci sont extraites du commentaire sur le Livre de la Sagesse, dont on trouve une édition annotée dans Théry (1928) et Théry (1929), où elles apparaissent respectivement dans Théry $(1929,353)$ et Théry $(1928,386)$. 
l'être et l'être, la vie. Ici, le platonisme est surmonté13 par le caractère développemental de la vie, par la vie de la copie qui s'approprie le modèle. À l'instar des citations précédentes d'Eckhart, les manières de voir aristotélicienne et platonicienne (c'est-à-dire les deux manières de penser fondamentales qui régissent la philosophie et la théologie) ne suffisent donc clairement pas pour comprendre ou pour critiquer ce qui est en jeu. Ce qu'il faut, c'est une manière de voir qui s'inscrit dans un horizon totalement différent.

Cependant, de ce qui précède, il me semble déjà évident que la vie dont il est ici question n'est pas une vie immédiate et sans intermédiaire, mais une vie que toutes sortes de négations ont fissurée et qui a subi des ébranlements telluriques /16/ — c'est la vie du mouvement dialectique. De surcroît, il me semble également évident qu'au bout du compte tous les aspects de ce mouvement dialectique correspondent à la vie. Même la négation de la vie est une autonégation de la vie à l'intérieur d'elle-même et, au fond de cette négation, réside l'autoaffirmation de la vie qui se nie. Et cette autoaffirmation est, en son fond, la manifestation de la force de la vie immédiate et sans intermédiaire comme forme primitive de la vie. Dans la négation de la négation se trouve la dynamis primitive qui impulse l'élan vers la vie. Cette forme primitive immédiate constitue l'assise qui supporte la négation dans l'autonégation; elle devient la force motrice de l'avancement dans la négation de la négation. En ce sens, dans le mouvement dialectique de la vie, même la médiation est, en son fond, immédiate. Le mouvement dialectique de la vie fait toujours de l'immédiat une médiation. La vie immédiate qui constitue le fond de ce mouvement est toujours le commencement en même temps que l'achèvement du mouvement. Le mouvement émerge toujours de là et s'achève toujours là. De ce fait, la négation qui est médiation et l'immédiat, en étant médiatisés, deviennent immédiats. C'est par la négation de la copie que le modèle se manifeste; et c'est par la négation du modèle que se manifeste la vie de la copie qui s'est approprié le modèle. Modèle et copie sont ici médiatisés, et la vie immédiate, où la vie du modèle et la vie de la copie constituent les deux aspects d'une même réalité, se fait alors jour. La vie de celui qui est juste n'est que par le fait qu'il accomplit la justice dans sa vie; la justice n'est que dans la vie de celui qui est juste. Dans ce cas, il est insuffisant de parler de l'union des deux. Les deux doivent être purement un. C'est ici que se manifeste le caractère

13. Ici, Nishitani utilise le vocabulaire eckhartien de la «percée» (突破), en le sortant de son contexte d'usage habituel. 
originaire de la vie, c'est-à-dire son absence de fondement et son absence de limite. Lorsqu'Eckhart affirme que la naissance de Dieu dans l'âme de l'homme désigne le pur un de Dieu et de l'homme, il faut le comprendre en supposant ce caractère originaire de la vie. Et alors, ce qu'Eckhart nomme l'un pur ou le néant apparaît comme le caractère originaire de la vie en tant que tel. S'il en va ainsi, dans quel sens faut-il le comprendre?

Le caractère originaire de la vie, l'absence de raison, l'affirmation absolue du "vivre seulement pour vivre ", voilà ce qui réside à la source de la vie partout où elle se meut; mais on ne peut pas dire, inversement, que tous les mouvements de la vie découlent de cette source. Le caractère originaire de la vie n'apparaît authentiquement que dans l'accomplissement du mouvement dialectique de la vie. "Vivre pour vivre », telle est aussi, par exemple, la devise de l'épicurien qui a cédé au sentiment que tout est égal /17/. Et même dans ce cas, il y a assurément quelque chose qui rappelle le caractère originaire de la vie. De même que, dans le désespoir, se manifeste la négation absolue de la vie, le renoncement à soi qui accompagne ce désespoir présente les traits déformés d'une affirmation absolue de la vie.

Dans le sentiment que tout est égal, pour reprendre cet exemple, l'ego en tant que forme est brisé. Parce qu'il se raccroche de toutes ses forces à lui-même, parce qu'il est attachement à soi, l'ego en tant que forme connait, dans le sentiment que tout est égal, une extase qui le laisse sens dessus dessous. En fait, nombreuses sont les personnes qui se sont ouvertes à la religion après avoir traversé ce sentiment que tout est égal. Mais bien qu'il effleure le caractère originaire de la vie et son absence de raison, le sentiment que tout est égal reste le propre d'une vie qui cherche hors d'ellemême une multitude de raisons éparpillées, le propre d'une vie qui se cache à elle-même le caractère originaire de la vie. Ainsi, le caractère originaire est, pour une telle vie, un simple vide; et elle se rend compte que son fondement ainsi que les raisons de vivre sont, au fond, vacuité. Afin d'éviter une telle prise de conscience de la vacuité, elle se cherche un fondement de-ci de-là et, ce faisant, elle s'érige en se raccrochant à des raisons éparpillées sur une vacuité foncière.

Cependant, dans la vie normale qui possède en elle-même une raison qui lui donne une certaine cohérence et qui, par elle, est unifiée, on trouve la situation inverse. (Dans ce cas, peu importe que les raisons de vivre soient innombrables en fonction de ce que chacun considère comme sa profession, son Beruf.) La vie normale diffère de celle qui s'abandonne au désespoir, elle possède son fondement en elle-même. La "raison de vivre » qu'elle se donne à elle-même lui procure un fondement. De plus, cette rai- 
son constitue sa finalité; elle la détermine par rapport au futur. Ce qui, au fond de la vie présente, la soutient, c'est ce qui la détermine en la rapportant constamment au futur. Ici apparaît une vie qui englobe le passé et le futur à partir du présent, et qui ainsi se coupe de la vie originaire, une vie qui s'enferme en elle-même. Et ce n'est rien d'autre que l'ego en tant que forme qui assure l'unité de cette vie refermée sur elle-même. Par ailleurs, bien que cette vie se tourne vers le futur et s'y prolonge indéfiniment, elle ne se sépare à aucun moment d'elle-même. Cet attachement à soi correspond à l'ego en tant que forme déterminée. C'est par cette forme que la vie est séparée de son caractère originaire. En revenant en elle-même, en devenant "pour soi ", en prenant conscience d'elle-même, la vie se sépare de sa propre source. Ici s'instaure la position de l'entendement, de l'ego compris comme une «substance» qui s'oppose à toutes les autres et s'en tient isolée. La vie, en se faisant face à elle-même, en vient simultanément à faire face aux autres; et c'est alors que se produit la distinction entre l'intérieur et l'extérieur $/ 18 /$.

À partir de là, on peut aussi comprendre pourquoi Bergson souligne, comme on le sait, le fait que sa "conscience réflexive", ou son "entendement ", partage sa source avec la représentation spatiale qui considère le flux de la vie à la manière des objets qui, discriminés, se juxtaposent dans l'espace. Ainsi émerge «l'âme» qui, au sein de la vie, a divorcé d'avec la vie. Malgré tout, cette âme aussi est vie, et même dans le divorce d'avec la source de la vie apparaît le caractère originaire de la vie. Ce qui atteste cela, ce n'est rien d'autre que la subjectivité, ou plutôt l'intentionnalité, qui s'instaure seulement avec «l'âme». La non-dualité du pour-soi et du pour autrui dans l'âme est ce qu'on appelle l'intentionnalité. Conjointement à l'instauration de «l'ego ", lequel vise l'autre en se distinguant lui-même de tout autre, apparaissent la subjectivité et son caractère absolument unique du point de vue noétique. Le caractère absolument unique du soi, parmi les étants innombrables qui existent depuis un passé infini et jusque dans un futur infini, est fondé sur la noèse absolue qui se produit au moment où il voit, entend et pense, sur le fait que personne ne peut voir, entendre ou penser à sa place. Et cette noèse est la manifestation de la source de la vie sous-jacente. Cependant, ici encore, la source de la vie se manifeste dans l'ego, mais on ne peut pas dire que l'ego soit à la source de la vie. En d'autres termes, l'ego, sans s'apercevoir de la manifestation de la source de la vie comme telle en lui-même, pense tirer de lui-même cette noèse. Cet aveuglement à propos du fait que l'ego n'est jamais à la source de la vie correspond précisément à l'émergence de l'ego en tant que forme détermi- 
née, de l'ego compris comme substance, c'est-à-dire de l'ego conçu à la manière d'une chose déjà noématisée. Si l'ego était à la source de la vie, cette forme serait, me semble-t-il, brisée de l'intérieur et la noèse absolue authentiquement conscientisée. Or, même si la source de la vie se manifeste à l'ego, même si, l'ego n'étant pas à la source de la vie, la noèse absolue apparaît quand il voit, entend et connaît, l'ego considère cela malgré tout comme l'acte de l'ego noématisé. Cette "réflexion» est au fondement de toute « réflexion» de ce type (c'est-à-dire de la « doxa » grecque) et constitue une idée fondatrice. C'est à partir de cette idée fondatrice que s'établit l'ego en tant que forme et que s'effectue le divorce d'avec le fondement de la vie. Dès lors, de quoi cela dépend-il que cette forme soit brisée et que l'on revienne au fondement de la vie? /19/

D'un côté, le soi compris comme substance est pour-soi et se connaît lui-même et, de l'autre, il s'oppose à toutes les autres choses et les voit de l'extérieur (objectivement). Ou encore, il se connaît lui-même de la même manière dont il a pris l'habitude de voir toutes choses objectivement (telles des choses juxtaposées). C'est là la signification que revêt l'entendement discriminant. Et la marque de cette opposition sujet-objet, de cette façon de voir depuis l'extérieur, c'est précisément la «représentation». La représentation est le contenu de l'acte de l'ego lorsque celui-ci, isolé, voit toutes les autres choses de l'extérieur (objectivement). Dans la manière de voir objectivement, quand on dit que l'ego voit une chose, la représentation vient s'interposer entre les deux et, cela, en dépit du fait que l'ego lui-même pense voir directement l'objet. Cela est emblématique de la fracture entre l'ego et la chose vue objectivement par l'ego. En s'interposant entre les deux, la représentation les tient éloignés l'un de l'autre. La représentation est la cataracte qui se produit dans l'œil qui voit tout objectivement; et voir à travers cette cataracte de la représentation empêche de voir directement depuis l'intérieur de l'autre, cela entrave l'intuition ou la «sympathie vivante» de Bergson. (Pour cette raison, on ne peut pas penser que l'ego et l'objet précèdent la représentation, laquelle se produirait à partir de leur mise en relation. L'ego vu comme substance et l'autre vu comme objet, d'un côté, et la représentation, de l'autre, se produisent simultanément à, et conjointement à, l'instauration de la manière de voir depuis l'extérieur.) Pourtant, non seulement la représentation s'interpose entre l'ego et l'objet et les tient séparés l'un de l'autre, mais en même temps elle les rapproche l'un de l'autre en étant leur médiation. En tant que représentante de l'objet, elle rend celui-ci manifeste à l'ego et, en tant que contenu de l'acte de l'ego, elle oriente celui-ci vers l'objet. Dans l'acte par lequel il fait de la représen- 
tation son contenu, l'ego se tourne vers l'objet, se rapporte à l'objet. La représentation correspond à la force d'attraction de l'objet qui attire l'ego. Par conséquent, pour briser l'ego en tant que forme et revenir à la source de la vie, il faut d'abord que la représentation soit extirpée de l'ego. L'ego compris comme forme se constitue en tant que non-dualité du pour-soi et du pour autrui. Dès lors, si le pour autrui est nié, alors le pour-soi l'est également. Si l'on renonce au rapport à l'objet, alors la forme déterminée de l'ego est également brisée et, en sens inverse, il se pourrait que le lien entre l'ego et la source de la vie apparaisse. Ainsi, ce qui est d'abord requis, c'est la négation du pour autrui, la suppression de la représentation. /20/

Chez Eckhart, une telle élimination de la représentation constitue également une condition préalable à la naissance du Fils de Dieu en l'âme. En accord avec la tradition depuis Aristote, il utilise le concept d'image (Bild). Lorsque l'âme extirpe hors d'elle-même toute image et devient une forme vide, alors y retentit la parole divine qui, en raison du grand bruit qui y régnait jusque-là, restait inaudible. En devenant un lieu vide, l'âme devient une matrice dotée d'une forme nouvelle. Devenir un lieu vide, c'est recevoir immédiatement la parole divine. Plutôt, c'est le fait que la parole de Dieu, présente en l'homme depuis le commencement éternel, lui apparaisse à ce moment. L'homme est créé dans le temps. Et cependant, il abrite quelque chose qui était avant que le monde, le temps et lui-même ne soient créés, à savoir l' «incréé » - un lien vivant avec Dieu, une union vitale. Le fond de son âme est, si l'on peut dire, percé par la pointe de la vie divine. Non, plutôt, cette pointe est précisément le fond même de l'âme. Et quand l'âme devient un lieu vide, elle rencontre cette pointe, la vie divine. Et cette manifestation de ce qui est antérieur à la création, ce qu'Eckhart nomme tantôt «fond de l'âme» ou "éclair » de la vie divine, tantôt "pauvreté en esprit », "paix» ou encore "silence» correspond précisément à la naissance de Dieu dans l'âme. C'est que l'âme y est à l'unisson avec Dieu et y connaît Dieu. Bien entendu, cette connaissance, parce qu'elle est ce qui s'instaure là où il n'y a aucune représentation ou « image ", n'est pas la connaissance raisonnée de l'entendement discriminant, mais un savoir intuitif. Si l'on appelle «savoir» le savoir qui véhicule des représentations, il s'agit alors ici au contraire d'un non-savoir. Pour cette raison, Eckhart nomme ce savoir intuitif «savoir du non-savoir ".

Cependant, Eckhart voit en ce lieu, qui était considéré jusque-là comme le plus achevé, un degré ultime qui doit encore être dépassé. Car, même si l'on dit que l'âme reçoit la parole divine en devenant un lieu vide et qu'elle rencontre la vie divine qui la perce, dans la mesure où l'on se 
contente d'une telle union, l'âme est à la fois unie à Dieu et distincte de lui, et le savoir intuitif renferme encore le double aspect de ce qui intuitionne et de ce qui est intuitionné. Dieu ne saurait être limité à ce qui est opposé objectivement, à ce qui est représenté, à ce qui est vu de l'extérieur au moyen d'une «image». Comme dans le cas de la cognition des choses dont il a été question précédemment, l'image de Dieu (imago Dei) dans l'âme, la parole divine (le Fils de Dieu), /21/ est ce qui, en rapprochant de l'extérieur l'âme vers Dieu, institue une séparation d'avec Dieu. L'âme y conserve son caractère de pour-soi, son attachement à soi, elle conserve sa détermination comme forme. Aussi la vie divine qui vient en elle ne peut-elle jamais venir en tant qu'authentiquement vivante. Si l'on reprend une métaphore que nous avons déjà utilisée, il s'agit de l'eau qui remplit seulement l'apparence déterminée du calice vide. Quand Dieu vient authentiquement en tant que Dieu, la forme de l'ego est brisée et l'eau de vie déborde du calice. Dans la mesure où l'ego se maintient en tant que forme, dans la mesure où il conserve la signification de lieu, Dieu est un Dieu pensé. "Quand nous intuitionnons, nous ne sommes pas un avec ce que nous intuitionnions. Car là où il n'y a rien d'autre que l'un, on ne voit rien. C'est pourquoi on ne peut voir Dieu que par la cécité, le connaître que par le non-savoir, le comprendre que par la déraison ${ }^{14}{ }$ (Büttner, II, p. 151). «Tant que l'âme a Dieu, connaît Dieu et sait quelque chose de Dieu, elle est séparée de Dieu ${ }^{15}$ » (II, p. 165). «L'homme doit devenir si pauvre qu'il ne soit pas un lieu où Dieu puisse œuvrer et qu'il n'abrite pas non plus en lui un tel lieu. Tant que l'homme conserve en lui un lieu, il conserve une différence ${ }^{16} »(\mathrm{I}$, p. 206). De ces passages, il ressort que ce qu'on appelle " union » signifie tout à la fois être uni et distinct. Dès lors, en quoi consiste le saut de l'union à l'un pur?

Que l'âme et Dieu ne font qu'un tout en se tenant pourtant l'un en face de l'autre, cela signifie qu'ils s'interpénètrent réciproquement. Cela signifie que Dieu brise la forme de l'ego et l'ego l'image de Dieu. Eckhart va

14. Sermon "Des obstacles à la vraie spiritualité », aussi intitulé: «Pourquoi l'âme doit rejeter hors d'elle tous les saints ». Nishitani altère la première phrase de cette citation. Pour la seconde, par contre, il est plus proche de l'original que les traductions françaises.

15. Sermon «Du royaume de Dieu».

16. Sermon 52 «De la pauvreté en esprit». Nishitani renvoie erronément à la p. 206, alors que cette citation est tirée de la page 185 . 
jusqu'à déclarer: "Je dois devenir Dieu et Dieu moi ${ }^{17}$ » (op. cit., I, p. 178). Une assertion de ce type, si on ne la réfléchit pas au moyen du mouvement dialectique de la négation réciproque, il me semble que l'on ne saurait la comprendre autrement que comme une pure folie. L'intuition, qui englobe ce qui voit et ce qui est vu, les deux yeux qui se font face, les deux regards qui se croisent, cela doit devenir un œil, un regard. "L'œil par lequel je vois Dieu est identique à l'œil par lequel Dieu me voit. Mon œil et l'œil de Dieu sont un œil et une vision ${ }^{18}$ » (Pf. 96).

Dans la perception visuelle de mon œil, se manifeste le caractère originaire de la vie en tant que mon unicité noétique absolue, en tant que ma vie antérieure à la création, en tant que ce que je "devais être" dès le commencement éternel. Or, dans la mesure où cette perception visuelle est conçue comme l'œuvre de l'ego en tant que forme qui a divorcé d'avec la source de la vie /22/, l'ego, par son statut de pour-soi, c'est-à-dire dans le fait même qu'il est ego, s'érige comme digue d'un lieu sans digue. Un tel statut de pour-soi est nécessaire pour qu'apparaissent cette noèse absolue, le caractère absolument unique du point de vue subjectif, c'est-à-dire précisément le caractère originaire de la vie; et pourtant, par lui, l'ego divorce en même temps de la source de la vie. Le caractère originaire de la vie dans l'ego, tout en se faisant jour de la manière la plus limpide, se dissimule le plus profondément. L'acte noétique de l'ego, bien qu'il soit une manifestation immédiate du caractère originaire de la vie, demeure encore une négation absolue de la source de la vie; il est une médiation négative absolue, vouée à refluer vers cette source. C'est-à-dire, comme je l'ai dit plus haut, qu'ici l'absence directe de médiation devient elle-même une médiation. C'est ainsi que, dans la mesure où l'ego est la négation absolue de la source de la vie, la source de la vie s'oppose à l'ego depuis l'au-delà de l'ego en tant qu'absolument autre. Et l'ego, par son égoïté même, élève une digue au sein de la vie et s'impose à lui-même des limites. Pour que l'ego remonte à la source de la vie, ces limites doivent être brisées depuis au-delà de celles-ci. (Dans ce contexte, il arrive qu'Eckhart puisse aussi parler de grâce divine, d'amour divin.) Et il faut que l'ego, en étant transpercé au plus profond par une nouvelle forme plus haute, ait la forme de son égoïté brisée. Pour utiliser une expression d'Eckhart, il doit subir un durchformen par une forme plus haute.

17. Sermon 83 «Du renouvellement dans l'esprit». Nishitani renvoie erronément à la p. 178 , alors que cette citation est tirée de la page 179 .

18. Sermon 12. Nishitani renvoie à l'ouvrage de Pfeiffer (1857). 
Cependant, pour que ce durchformen soit authentiquement durchformen, ce qui forme et ce qui est formé ne sauraient être distincts. Une forme plus haute, cela signifie un mode de vie plus haut de l'ego et il doit s'agir d'un nouvel ego. Que l'ego ait sa forme brisée et soit recomposé sous une nouvelle forme, cela correspond à une métamorphose de l'ego. Cela signifie qu'une nouvelle vie, jaillissant de l'intérieur de l'ego, transforme celuici. Ou encore, cette nouvelle vie qui déborde les limites que l'ego s'est imposées à lui-même, qui le brise et le submerge, se manifeste depuis l'intérieur de l'ego. Cette vie excède les limites et s'approprie la forme plus haute qui est au-delà. Si l'on emprunte une autre expression à Eckhart, il s'agit de l'überformen. Cela signifie excéder les limites; cela signifie que l'au-delà devient ce monde-ci. C'est seulement dans un tel durchformen-commeüberformen que l'on peut affirmer que «l'œil de Dieu et mon œil sont un œil et une vision » et que la source de la vie manifeste sa noèse absolue /23/ sous sa forme véritable. "Je dois devenir Dieu et Dieu moi. Ce "il" et ce “je” doivent devenir un et être un et, dans cet "être”, ils doivent œuvrer à une même œuvre (ein werc wirkent $)^{19}$ " (I, p. 178).

Dans cette dernière citation apparaît clairement la raison pour laquelle j'ai dit plus haut qu'en considérant l'être comme vie, Eckhart a dépassé l'aristotélisme (le thomisme) et plus encore le platonisme (l'augustinisme): considérer l'être comme vie revient, d'une certaine manière, à dépasser l'être. Bien entendu, dans la mesure où l'être au sens courant, mais aussi l'être des idées, voire l'être de Dieu se posent comme objets, ils empêchent que se produise l'œuvre pure. Comme l'a démontré Bergson de manière très précise, déterminer la vie au moyen de l'être signifie la mort de la vie. L'œuvre pure doit dépasser même l'être de Dieu, s'en détourner pour revenir aux idées et, davantage encore, revenir à l'être des choses sensibles. L'être des choses sensibles est le reflet des idées; les idées sont la véritable réalité, un être d'un ordre supérieur. Cependant, si on se place du point de vue de l'œuvre pure qui est au-delà de l'être, même les idées, en dépit du fait qu'elles sont la véritable réalité (plutôt, parce qu'elles sont la véritable réalité), sont une "apparence " modélisée sur fond de vacuité, un reflet esquissé d'après la vacuité; et c'est comme telles qu'elles sont la véritable réalité. Quant aux choses sensibles, en dépit du fait qu'elles sont, en tant que reflets des idées, éphémères à la manière de reflets de reflets, elles

19. Sermon 83 «Du renouvellement dans l'esprit». Nishitani renvoie erronément à la p. 178 , alors que cette citation est tirée de la page 179 . 
constituent la réalité concrète qui est au-delà de la pure "apparence »; elles sont également la véritable réalité, mais en un sens différent.

Il me semble qu'ici s'ouvre une voie par laquelle platonisme et aristotélisme, en étant dépassés, sont ravivés. Mais pour cela, la source de la vie doit être le néant de tout être. Lorsque l'antagonisme entre Dieu et l'homme est dépassé noétiquement, tant l'être de Dieu que celui de l'homme sont dépassés. En dépassant l'être de Dieu au fond de Dieu (Eckhart l'appelle "déité ») et en dépassant l'être du soi au fond du soi (l'ego en tant que substance), Dieu et l'homme deviennent un pur un dans le néant et opèrent une seule œuvre. L'homme, en se fondant sur l'abîme qui a dépassé même l'être de Dieu, c'est-à-dire en revenant le plus profondément à Dieu, revient authentiquement à soi, devient un soi qui agit, un soi libre. Eckhart déclare: «Le fond de Dieu est mon fond, mon fond le fond de Dieu ${ }^{20}$ » /24/ (I, p. 127). Par conséquent, lorsque l'œil de Dieu et mon œil deviennent un œil et une vision, "l'ici et maintenant » où se produit l'acte de voir précède la création divine, il est l'au-delà de l'être de Dieu. Là où l'on dépasse l'antagonisme entre l'au-delà et ce monde-ci, l'au-delà de l'au-delà devient la mondanéité de ce monde-ci. C'est la raison pour laquelle Eckhart dit que l'homme devient libre dans le néant de Dieu, ou encore que l'homme est libre en se fondant sur "l'abîme sans fond " qui constitue son propre fond. Bergson, qui avait entamé sa carrière philosophique avec l'examen du problème de la liberté, en est finalement lui aussi arrivé à une théorie de la religion à propos de l'œuvre créative qui, en devenant un avec l'acte de création de Dieu, prolonge ce dernier.

\section{Vie religieuse et esprit positif}

Dans ce qui précède, j'ai tenté de dégager ce qu'il me semble être l'inspiration fondamentale commune au Zarathoustra de Nietzsche et à Maître Eckhart, sans qu'il y ait de rapport entre leurs doctrines respectives. Je ne cherche pas à affirmer par là que leurs doctrines relèveraient des mêmes catégories philosophiques. Et je n'oublie pas que, derrière les figures presque diamétralement opposées du moine prêcheur qui a vécu en monastère à la charnière des XIII ${ }^{e}$ et XIV ${ }^{e}$ siècles et du sage orgueilleux qui, à la fin du XIx ${ }^{e}$ siècle, a explicité la volonté de puissance qui portait la marque de l'athéisme, il y a deux époques aux atmosphères complètement différentes et que la forme des questions qui se posaient alors ainsi que les réponses

20. Sermon $5 \mathrm{~b} \ll$ Du fils $»$. 
qui y furent apportées diffèrent radicalement. On pourrait même voir chez Eckhart un des esprits les plus médiévaux qui soient et chez Nietzsche un des esprits les plus modernes qui soient.

Pourtant, en dépit d'un tel éloignement de leurs figures historiques, je ne peux m'empêcher de constater un élément commun au fond de leurs pensées respectives. Ne pourrait-on pas considérer que, contre toute attente, ils se sont rencontrés à l'apogée de la vie (ou à sa source), l'un par son effort de mener l'esprit à une liberté sans obstacles en traversant la complexité de la connaissance théologique, l'autre par sa critique stricte et radicale à l'encontre de l'esprit de la culture moderne et par sa tentative de sortir du chaos qui habitait celle-ci /25/?

L'attitude fondamentale qui leur est commune est l'approfondissement du mouvement dialectique de la vie. C'est l'approfondissement de l'affirmation de l'homme à travers sa négation. Chez nos deux penseurs, l'approfondissement de l'affirmation de l'homme se manifeste là où l'alliance (voire l'union) avec Dieu est envisagée comme un stade intermédiaire qu'il faut encore surmonter. L'alliance ou l'union avec Dieu, cela correspond encore à être à la fois unis et distincts. Dans la mesure où l'alliance ou l'union implique une distinction, c'est-à-dire dans la mesure où elle représente Dieu opposé à Dieu et considère Dieu comme un objet, il y persiste la mort, la négation de la vie, le divorce d'avec la réalité ultime de la vie.

La réalité ultime de la vie correspond, chez l'un, à "l'ici et maintenant » et, chez l'autre, à l'abîme sans fond de la vie. Mais, chez le premier, cette réalité ultime se dissimule derrière le soi qui s'oppose à Dieu et, chez le second, elle se dissimule derrière Dieu qui s'oppose au soi. Même l'alliance ou l'union se fonde déjà sur le fait que ces "derrières » seraient purement un; mais dans la mesure où ces «derrières» se distinguent l'un de l'autre et se dissimulent mutuellement, ils sont encore deux. Lorsqu'on surmonte cette situation, les deux «derrières » deviennent un «devant ". Devant Dieu et devant le soi deviennent un. Cette noèse absolue, Eckhart l'exprime comme «le fond de Dieu [qui] est mon fond, mon fond le fond de Dieu ", comme "l'œil de Dieu et mon œil [qui] sont un", ou encore comme la liberté qui se fonde sur le néant; Nietzsche l'exprime comme "sommet et abîme [qui] maintenant sont confondus ", comme "le midi et le minuit [qui] sont devenus un ", ou encore comme le trop-plein de vie qui déborde l'ego, comme l'activité créatrice de l'homme sans Dieu.

Quand Eckhart déclare: «Lorsque je veux me tenir pur dans la volonté de Dieu et aussi lorsque je veux me tenir libre de la volonté de Dieu, de toutes les œuvres de Dieu et de Dieu lui-même, alors je transcende toutes 
les créatures et je ne suis ni Dieu ni créature ${ }^{21} »(I, p .185)$ et quand Nietzsche déclare: "La volonté d'engendrer m'attira loin de Dieu et des dieux; qu'y aurait-il donc à créer s'il y avait des dieux? » (cité précédemment), ils se tiennent tous deux là où la profondeur insondable de la vie se consume dans l'instant présent ou, pour ainsi dire, ils se tiennent dans la vie de la vie. Pour l'homme, dans la mesure où, opposé à Dieu, il est une créature, il n'y a pas de vie, c'est-à-dire pas de création. "Sommet et abîme, — tous deux maintenant sont confondus. [...] Il faut que ce soit maintenant ton courage le meilleur de n'avoir plus de chemin derrière toi! /26/ [...] Ton pied lui-même effaça derrière toi le chemin" (Zarath., III. "Le voyageur»).

Pourtant, cet approfondissement de l'affirmation constitue un approfondissement de la négation. L'homme doit devenir une fois entièrement "créature". Nietzsche affirme même: "Dire sans cesse "Oui", c'est ce qu'ont appris les ânes et ceux qui ont leur esprit» (Zarath., III. «De l'esprit de pesanteur »). La vie en vient à élucider le soi en niant le soi. Ici se trouve la voie de la connaissance en son sens authentique, c'est-à-dire en son sens philosophique. Contrairement à ce que l'on pense généralement, Nietzsche ne proclame pas simplement la volonté ou la vie immédiate. La vie ou la volonté dont il est ici question, c'est la vie qui en vient à être purifiée par la connaissance stricte qui nie, dans le soi, ce qui est trop humain. Il arrivait souvent à Nietzsche de parler avec plaisir de la connaissance comprise en ce sens. Ainsi, il a par exemple déclaré: «Pour moi vous êtes des tièdes, mais froid est le flot de toute profonde connaissance. Froides comme glace sont les sources les plus intimes de l'esprit: pour des brûlantes mains et des actions brûlantes on les nomme réconfort» (II. "Des sages illustres»).

Même s'il avait largement pris ses distances d'avec Thomas d'Aquin, Eckhart s'opposa aux volontaristes de l'époque, qui refusaient l'intellectualisme thomiste, et insista sur l'intellect et la connaissance. Ce qu'il appelle "savoir du non-savoir ", et qui se manifeste à la base de l'élimination de toute Bild et de tout savoir objectifiant, exprime, à travers l'approfondissement de l'intellect, qui est la dimension négative de la vie, le fait que la vie elle-même s'approfondisse et que, ultimement, la négation se change en affirmation comme telle. (Ce qui, chez Eckhart, correspond à l'élimination de la représentation, le Nietzsche dyspepsique le symbolise par le dégoût: «Ekel! Ekel! Ekel!", s'écrie Zarathoustra $\left.{ }^{22}\right)$. Ce change-

21. Sermon 52 «De la pauvreté en esprit».

22. Dans Ainsi parlait Zarathoustra (Livre III, "Le convalescent»). 
ment en affirmation est aussi foncier que l'était la négation. La noèse absolue qui apparaît ici, l'acte de la création libre, acquiert sa réalité la plus haute dans l'action corporelle. En ce sens, le corps est l'âme la plus profonde, une âme plus profonde même que l'âme. L' "âme", l'esprit ne constituent qu'une part de toute la vie qui est concentrée et condensée dans le corps. Le corps les excède et est appuyé par l'abîme sans fond de la vie. Il y a ce mot d'Eckhart: "Dieu a établi l'âme dans la libre détermination d'elle-même. Ainsi, /27/ Dieu ne peut rien imposer à l'âme qui soit audessus de sa volonté libre ni exiger quoi que ce soit que l'âme ne veut pas. Par conséquent, ce qu'elle choisit dans ce corps par sa propre liberté, l'âme peut s'y tenir ${ }^{23} »(I$, p. 197).

Concernant l'insistance nietzschéenne sur le corps, il ne me semble pas nécessaire d'en donner ici une explication détaillée. Elle apparaît déjà clairement dans les passages suivants: "Je suis corps et âme", voilà ce que dit l'enfant. Et pourquoi ne devrait-on pas parler comme les enfants? Mais celui qui est éveillé, celui qui sait, dit: "Je suis corps de part en part, et rien hors cela"; et l'âme ce n'est qu'un mot pour quelque chose qui appartient au corps "; "Le corps est une grande raison »; " "Moi", dis-tu, et tu es fier de ce mot. Mais ce qui est bien plus grand ${ }^{24}$ - ton corps et sa grande raison: il ne dit pas "moi", mais il le fait» (Zarath., I. "Des contempteurs du corps »).

Je ne pense pas que l'on puisse considérer comme purement et simplement fortuits les traits communs fondamentaux qui caractérisent l'inspiration de ces deux penseurs, tels que ceux que j’ai évoqués ci-dessus. Les conditions historiques dans lesquelles l'un et l'autre évoluèrent, si elles semblent à première vue différer complètement, ne présenteraient-elles pas déjà quelque caractéristique fondamentale commune ? Pour résumer schématiquement, les époques qui suivirent la mort de Thomas d'Aquin et celle de Hegel furent marquées par de grands bouleversements qui se propagèrent, depuis les profondeurs de l'histoire, à tous les domaines de la culture.

La confusion qui régna dans le monde intellectuel après la mort de Thomas d'Aquin avait une cause profonde et ancienne. Il me semble que l'on peut faire remonter son origine à l'antagonisme entre la révélation surnaturelle, qui fut de plus en plus remise en question au fil du dévelop-

23. Sermon «De la colère de l'âme et de son vrai lieu». Nishitani renvoie erronément à la p. 197, alors que cette citation est tirée de la page 196.

24. Nishitani omet: ", en quoi tu ne veux pas croire». 
pement de l'histoire de la pensée médiévale, et la raison naturelle, entre la foi et la connaissance. Cet antagonisme a toujours habité les études chrétiennes depuis que les Pères de l'Église grecs, et en particulier Augustin ${ }^{25}$, ont souscrit à la philosophie grecque. Non, d'une manière plus radicale, on pourrait aller jusqu'à affirmer que cet antagonisme est aussi ancien que l'éveil de l'intelligence humaine et que, sous la pression de cet éveil au seuil de l'ère historique de l'humanité, il a commencé à se manifester conjointement à l'effondrement des religions primitives et, par conséquent aussi, des sociétés primitives. Après Augustin, la scolastique, qui s'est épanouie dans le prolongement de la longue histoire de la christianisation des peuples germains, constitua une excroissance de la pensée grecque à l'intérieur du système doctrinal du christianisme et, dès lors, l'antagonisme entre les deux /28/ - l'antagonisme entre ces attitudes humaines complètement différentes - commença à apparaître en plein jour. Ce fut le cas en particulier aux $\mathrm{XII}^{\mathrm{e}}$ et $\mathrm{XIII}^{\mathrm{e}}$ siècles lors de la propagation, dans les régions de culture chrétienne, de la philosophie aristotélicienne, réputée pour avoir déjà provoqué, deux siècles auparavant, de grands bouleversements dans le monde islamique. Au gré de la diffusion des écrits d'Aristote et des commentaires d'Averroès, l'averroïsme, qui avait aboli la distinction entre révélation surnaturelle et raison naturelle, et qui sera accueilli avec enthousiasme par les penseurs séduits par les Lumières, connut à l'époque de Thomas d'Aquin une grande popularité au sein de l'université de Paris. C'est ainsi que le monde intellectuel de l'époque s'apparentait à un enchevêtrement où se mêlaient la foi de la révélation chrétienne, l'aristotélisme d'Averroès qui s'appuyait sur la spéculation de la raison naturelle, l'augustinisme enserré par leur antagonisme, etc.

Le platonisme d'Augustin ne pouvait plus réconcilier cet antagonisme. D’après Augustin, les idées éternelles (rationes) constituent la véritable réalité et la raison humaine acquiert la connaissance vraie en intuitionnant, dans le monde des choses sensibles, les idées qui sont les archétypes de celles-ci. C'est alors que la lumière de l'intelligence divine se déverse dans la raison humaine; la raison est éclairée par cette lumière et c'est grâce à celle-ci et en celle-ci seulement qu'elle peut intuitionner les idées. Or, il va sans dire que l'affirmation selon laquelle on intuitionne ainsi toute chose grâce à la lumière divine dans les idées éternelles laisse entrevoir une profonde rupture d'avec l'esprit positif en tant que connaissance objective des

25. Nishitani compte erronément Augustin parmi les Pères grecs alors qu'il est en réalité l'un des quatre Pères de l'Église latine. 
choses sensibles (et, dans la modernité, en tant que connaissance scientifique), une profonde rupture d'avec la conscientisation de l'autonomie de l'intellect humain que véhicule cet esprit positif. La critique d'Aristote contre le platonisme découlait déjà du même constat; mais cet antagonisme, qui s'accentua progressivement au Moyen Âge, a atteint son paroxysme avec l'introduction de l'averrö̈sme.

C'est le grand mérite de Thomas d'Aquin que d'avoir donné un ordre et une cohérence à ce chaos intellectuel. D'un côté, en adoptant l'aristotélisme et en corrigeant ou réinterprétant l'enseignement d'Augustin, il a reconnu l'autonomie relative de la raison naturelle: la raison naturelle n'acquiert pas la connaissance par un déversement direct de la lumière divine dans l'esprit humain ou par un contact immédiat avec la lumière divine; au moment où l'homme est créé, la loi ultime de la pensée est inculquée à sa raison et l'habite; et, grâce à cela, la raison peut accéder à la connaissance des choses sensibles. D'un autre côté, il a assurément établi les limites de la connaissance naturelle: celle-ci ne peut pas s'élever audessus de la connaissance des choses sensibles /29/. Au-delà de ces limites, il reconnaissait le monde de la révélation. De la sorte, en même temps qu'il renversait l'averroïsme, il restaurait le platonisme d'Augustin.

Pourtant, cette prodigieuse synthèse, ce système harmonieux, que l'on peut considérer comme l'aboutissement de la pensée médiévale, ne put endiguer la profusion anarchique de courants d'idées qui se développèrent en tous sens. Ce qu'il faut en particulier observer, c'est que, en réaction aux symptômes de décadence des écoles scolastiques qui rivalisaient d'habileté dans l'analyse des concepts et se faisaient concurrence, de nombreux groupes de pensée religieuse libre se constituèrent, se répandirent rapidement, sombrèrent bientôt dans la dépravation de la pensée et la corruption de la morale et rendirent plus sombre encore le chaos intellectuel. Dans de telles conditions, il est naturel que, d'un côté, on s'efforçât de se raccrocher encore à la longue tradition de la pensée et que, de l'autre, on s'évertuât à accomplir l'idéal d'une vie libre. Car seule la vie est immédiatement réelle et elle seule, en même temps, est susceptible d'induire la négation de toute chose et de l'approfondir sans cesse. Et, comme je l'ai expliqué, l'aristotélisme qu'avait adopté Thomas d'Aquin offrait une telle possibilité. En accentuant cela, Eckhart a dépassé l'aristotélisme; et c'est ainsi qu'il a balayé les limites établies par Thomas d'Aquin et qu'il a, en fin de compte, dépassé même le platonisme. Dans ce retour à la réalité spontanée de la vie, se manifestent l'effondrement des choses anciennes et un départ vers des choses nouvelles. 
La plupart des gens s'accordent sur le fait que, aux alentours de la moitié du XIX ${ }^{\mathrm{e}}$ siècle, des bouleversements profonds se sont produits dans la vie spirituelle en Europe. L'époque actuelle continue de subir ces bouleversements et il me semble qu'il en sera ainsi jusque dans un futur assez lointain. En expérimentant d'une manière nouvelle et des plus profondes ces bouleversements, Nietzsche projeta leur ombre au loin dans le futur. Je pense qu'il y a une similarité remarquable entre le monde intellectuel après la mort de Hegel et celui consécutif à la mort de Thomas d'Aquin. Cependant, ce sur quoi je voudrais surtout insister, c'est sur la question de savoir s'il n'y aurait pas entre les deux un rapport du point de vue, si l'on peut dire, de l'histoire intellectuelle mondiale.

La foi et le savoir, qui avaient été un temps conciliés chez Thomas d'Aquin, furent totalement scindés lors du tournant du Moyen Âge aux temps modernes. D'un côté, la réforme religieuse et, de l'autre, l'essor soudain de l'humanisme et des sciences naturelles ainsi que la logique de Bacon /30/ témoignent de cette scission. (Et ils s'inscrivent directement dans le prolongement de différents courants de pensée du Moyen Âge. Par exemple, le lien entre l'averroïsme et l'humanisme est analogue à l'influence de la science arabe sur la science européenne.) L'humanisme et les sciences naturelles ont provoqué des changements décisifs dans la vision religieuse de l'histoire et de la nature qui avait prédominé au Moyen Âge. Un fossé profond s'est progressivement creusé entre la vision religieuse du monde qui s'était maintenue jusque dans l'époque moderne et la vision du monde que défendaient l'humanisme et les sciences naturelles. Bien que Descartes, Leibniz et d'autres aient cherché à le combler, ce fossé s'est transformé, au XVIII ${ }^{\mathrm{e}}$ siècle, en ébranlement tellurique chez les penseurs des Lumières issus des penseurs libres. C'est alors que se firent les adieux définitifs au Moyen Âge.

On sait que c'est Kant qui chercha à réordonner de fond en comble et à réunifier le chaos hérité du Moyen Âge. Chez lui, on voit fusionner l'esprit du protestantisme, le mode de pensée de la science naturelle de Newton, l'empirisme depuis Bacon, le rationalisme depuis Descartes. Comme chez Thomas d'Aquin, ce qui assurait leur cohérence était la volonté d'attacher des limites au savoir afin de préserver une place pour la foi. Par ailleurs, comme l'explique Kant dans la dialectique de la première Critique, son époque avait souscrit, dans la sphère du savoir, à la théorie des catégories d'Aristote et à l'importance accordée au sensible et avait adopté les conceptions du platonisme dans la sphère de la morale supra- 
sensible. Cela atteste du fait que la pensée moderne est habitée par le même problème que la pensée médiévale.

Pourtant, en dépit d'une telle coïncidence formelle, ce qui distingue fondamentalement Kant de Thomas d'Aquin réside dans sa «révolution copernicienne». Celle-ci témoigne de la direction dans laquelle ce problème commun s'était développé, du Moyen Âge aux temps modernes. Le développement des sciences naturelles au cours des temps modernes, suivi du développement des sciences humaines, consolida immanquablement l'esprit positif et l'autonomie de la raison naturelle de l'homme. Ce qui posait alors problème, ce n'était pas chacun des résultats de la science. Ceux-ci sont susceptibles d'être très variables. Par contre, que l'esprit même de l'investigation scientifique, c'est-à-dire la conscientisation de l'autonomie de la raison naturelle, soit changeant, cela n'était pas pris en considération. (La plupart des chercheurs qui examinent les rapports entre science et religion, entre culture moderne et religion, etc., négligent ce fait.) La Critique de la raison pure constitua la déclaration philosophique de cette conscientisation de l'autonomie qu'avait acquise la raison naturelle. En se joignant au protestantisme, cette même conscientisation de l'autonomie de la raison /31/ s'étendit à la morale. L'autonomie de la volonté, qui est exposée dans La Critique de la raison pratique, a rendu hétéronome jusqu'au fait même d'accomplir la morale en tant que commandement divin.

Tous ces éléments, qui n'apparaissaient pas complètement chez Thomas d'Aquin, ont reçu, dans la philosophie kantienne, une importance considérable en tant qu'expression profonde de l'esprit moderne. Or, c'est précisément pour cette raison que le fait d'accorder une place à la foi était devenu plus problématique encore. Son examen des exigences de la raison pratique et sa théorie de la religion montrent combien cette entreprise d'accorder une place à la foi restait fragile. En posant des conditions supplémentaires aux Lumières anglaises et françaises, Kant les a en fait renforcées, ou plutôt il les a élevées. À sa suite, l'idéalisme allemand a poursuivi dans cette voie. Chez Kant, la raison naturelle était restée tolérante vis-à-vis du monde de la foi qui, bien qu'affaibli, continuait de se maintenir. De ce fait, elle cherchait à transformer ce qui était jusque-là donné comme contenu de la foi en contenu de la raison naturelle et à le réattribuer à cette dernière.

On peut avancer que ceci constitua l'ultime grande tentative de sauver le contenu de la foi, à une époque où était établie l'indépendance de la raison naturelle. Ainsi, après la mort de Hegel, lorsque les sciences natu- 
relles et les sciences humaines (l'histoire et les études sociales), qui n'avaient cessé de se développer entretemps, s'imposèrent en réaction à la philosophie naturelle de Schelling et de Hegel (ou en réaction à leur philosophie de l'histoire et à leur philosophie de la société), la foi devint complètement impuissante, et alors commença une période de désintérêt généralisé à l'endroit de la religion. Cela signifie qu'après le bref contrecoup que lui avait infligé Kant, le mouvement des Lumières du XviII ${ }^{\mathrm{e}}$ siècle commença à obtenir des résultats à partir du milieu du XIx ${ }^{e}$ siècle. En effet, le mouvement des Lumières avait été une bataille contre la vision religieuse du monde, mais cette bataille était désormais devenue caduque.

Et pourtant, tandis que se querellaient toutes sortes de matérialismes et d'idéalismes, un sens profond de la vie resurgit chez certains. L'évolutionnisme de Darwin constitua le moment le plus décisif pour ce nouveau surgissement. Leur sensibilité vis-à-vis de la vie différait de celle qui avait prévalu après la mort de Thomas d'Aquin en ceci qu'elle avait pris une dimension biologique, psychologique. Néanmoins, ils saisirent en profondeur que la vie, qui pénètre le soi et autrui de part en part, était à la fois ce qu'il y a de plus réel et de plus originaire. Il me semble que cette époque se distingue comme celle d'une "philosophie de la vie" au sens large. Dotés au plus haut degré d'une telle sensibilité à la vie, Nietzsche et Bergson furent emblématiques de la pensée occidentale de leur temps. Bien qu'ils soient partis d'un aspect différent de la vie, tous deux ont finalement approché la même position que celle à laquelle était arrivé Eckhart /32/.

Ce qui a entrainé l'émergence des synthèses harmonieuses de Thomas d'Aquin et de Kant, c'est le chaos qu'avait provoqué le développement de l'autonomie de la raison humaine, laquelle s'est rapidement imposée sur le devant de la scène de l'histoire humaine. Or, son développement s'est poursuivi jusqu'à renverser même ces tentatives d'harmonie, et elle en arrive à proclamer toujours plus fort son inéluctabilité. Jadis, cette autonomie était considérée comme la marque de la faute la plus profonde de l'homme, de son "péché originel ». Aujourd'hui encore, la plupart des théologiens pensent ainsi. Or, si cela relève assurément d'une profonde lucidité, n'est-ce pas un peu court pour l'homme moderne dont l'esprit a pris conscience de lui-même dans la connaissance scientifique et l'autonomie morale? Le goût du péché originel, quel scientifique, quel technicien le perçoivent-ils encore dans leur travail ? Quelle personne, allumant une lampe ou prenant un médicament, le perçoit-elle encore dans son acte?

Eckhart et Nietzsche sont apparus à des époques profondément en crise qui ont suivi des tentatives de conciliation. En acceptant de suivre 
l'orientation fondamentale du cours de l'histoire humaine et en œuvrant à l'autonomie de l'homme à travers la dialectique de la vie, ils se sont efforcés de surmonter l'antagonisme entre la foi et le savoir, et de résoudre la crise qui en a découlé.

\section{Références}

Büttner, H. (1919), Meister Eckeharts. Schriften und Predigten, I, Iena, Eugen Diederichs.

BütTner, H. (1919), Meister Eckeharts. Schriften und Predigten, II, Iena, Eugen Diederichs.

Heisig, J. (2008) [américain 2001], Les philosophes du néant / trad. par S. Isaac, B. Stevens et J. Tremblay, Paris, Cerf (Passages).

IsHIHARA, K. (1938), dir., 『哲学及び宗教と其歴史一波多野精一先生献呈 論文集』(La philosophie et la religion, et leur histoire. Recueil offert au professeur Hatano Seiichi), Tōkyō, Iwanami Shoten.

Oltmanns, K. (1935), Meister Eckhart, Francfort sur Main, Klostermann.

Pfeiffer, F. (1857), Deutsche Mystiker des Vierzehnten Jahrhunderts, vol. 2: Meister Eckhart, Leipzig, G.J. Góschen.

Théry, G. (1928), «Le Commentaire de Maître Eckhart sur le livre de la Sagesse", Archives d'histoire doctrinale et littéraire du Moyen-Âge, 3, p. 321-443.

- (1929), "Le Commentaire de Maître Eckhart sur le livre de la Sagesse (fin) ", Archives d'histoire doctrinale et littéraire du Moyen-Âge, 4, p. 233-394.

\section{Résumé}

Les pensées de Nietzsche et de Maître Eckhart constituent deux influences majeures qui traversent toute l'œuvre de Nishitani. Dans ce beau texte, rédigé lors de son séjour d'études (1937-1939) à l'université de Fribourg auprès de Heidegger, Nishitani fait une lecture tout à fait originale et innovante de la mystique eckhartienne, en la mettant en contraste avec la figure de Zarathoustra chez Nietzsche. Au-delà de leurs conditions historiques et de la spécificité de leurs démarches respectives, il leur reconnaît une attitude fondamentale commune qui consiste à approfondir ce qu'il nomme le «mouvement dialectique de la vie» (lequel vise une affirmation de l'homme à travers la négation de celui-ci) en direction du fond infondé qui soutient toute existence. Par «caractère origi- 
naire de la vie ", il entend la source de la vie dans son absence de fondement et de limite, dans son absence de raison. Ce faisant, et bien que relativement précoce (1938), cet essai annonce quelques-unes des intuitions les plus fulgurantes de Nishitani et offre une première esquisse de la plupart des thématiques qui lui tiendront particulièrement à cœur dans ses travaux à venir. Aussi y est-il déjà question de la vacuité, du savoir du non-avoir par opposition à la distinction sujet-objet, de la problématique de la représentation attachée à son exigence d'objectivité, de la compréhension de l'au-delà comme immanent à ce monde-ci, etc. Même la thématique du nihilisme, qui n'est pourtant pas nommément mentionnée, y fait écho dans l'exemple significatif du sentiment que tout est égal, que tout se vaut. En raison de la forme encore largement germinale que prennent ici ces réflexions, il est plus aisé d'en percevoir la portée et les imbrications. D'une certaine manière, on pourrait considérer cet essai comme un prélude aux importants développements que connaîtra la pensée nishitanienne jusqu'à l'ouvrage de maturité, Qu'est-ce que la religion?(『宗教とはなにか』) de 1961.

\section{Abstract}

The influence of Nietzsche's and Eckhart's thoughts permeates through all the work of Nishitani. In this beautiful piece of work, written during his research stay (from 1937 to 1939) at the University of Freiburg under the guidance of Heidegger, Nishitani gives a truly original and refreshing interpretation of Eckhart's mystic, contrasting it with the figure of Zarathustra in Nietzsche. Beyond their respective historical backgrounds and own agendas, he finds in them the same fundamental attitude, that is the deepening of what he terms the "dialectical movement of Life " (which consists in the affirmation of human through his negation) in the direction of the groundless ground sustaining all existence. By "elemental nature of Life», he means the original spring of life, which is groundless and limitless, which is without reason. Even though this is an early essay (1938), it points to some of Nishitani's deepest insights, and outlines most of the topics to which he will later go back again and again: emptiness, knowledge of non-knowledge (as opposed to the dichotomy of subject-object), objectivity linked to the intellectual representation, far side understood as near side, and so on. Even the topic of nibilism, though not namely mentioned, is present behind the illustration of the feeling that it all comes to the same. Owing to the fact that these reflections still have the simplicity and clarity of the first formulation, it is much easier to understand their implications and connections. In a way, this essay might be seen as a prelude to the tremendous developments that nishitanian thought will undergo until the masterwork of 1961, Religion and Nothingness (『宗教とはなにか』). 